\title{
Analysis of diagnostic methods and functional state monitoring of power oil transformers in electrical substations
}

\author{
Anton Lankin ${ }^{1, *}$, Alexey Baklanov ${ }^{1}$, and Igor Lankin ${ }^{1}$ \\ ${ }^{1}$ Platov South-Russia State Polytechnic University (NPI), 132 Prosvesheniya ul., Novocherkassk \\ 346428, Russia
}

\begin{abstract}
In this paper, we analyze the methods of diagnosis and control the functional state of power oil transformers in electrical substations. Oil transformers are the most efficient converters of electrical energy, which is reflected in a higher efficiency in comparison with dry transformers. Oil transformers are designed to work with power grids in large industrial complexes where energy conversion is required to protect equipment from sudden voltage surges. The high loads associated with this work require timely diagnosis and maintenance of transformers. The article discusses the most commonly used methods for diagnosing deviations of the technical parameters of electrical substations in the process of their operation. The authors proposed a method of impedance series-parallel identification, which allows not only to determine the equivalent circuit of the power oil transformer, but also to diagnose possible malfunctions. Functional circuits of the device for impedance series-parallel identification of faults in power oil transformers have been developed.
\end{abstract}

\section{Introduction}

As known, heating a power transformer during its operation is due to the presence of active losses in the windings and core. With the high power of the transformers, impressive heat losses occur. To prevent overheating of the transformer and violation of the insulation of the coil windings, oil cooling systems are used. When the quality of transformer oil deteriorates, active losses increase and the efficiency of the power device decreases, which negatively affects the process of converting electrical energy. Based on this, it can be concluded that the quality of the oil directly affects the durability of the power transformer. Thus, the most important functional elements, from the point of view of control, are transformer oil and the coil of the power device.

\section{Methods}

After delving into the existing approaches in the diagnosis and control of power oil

* Corresponding author: lankinjohn@yandex.ru 
transformers $[1,2,3,4,5,6,7,8,9,10,11,12]$, we can conclude that the approaches currently used are based on vibroacoustic, frequency and energy analysis $[13,14,15]$. If necessary, assess the condition of the cooling oil in power transformers using chemical analysis methods. Thus, it is worth noting that for the diagnosis and control of electrical systems of electrical substations they do not use a comprehensive analysis of many physical processes (mechanical, thermal, energy, magnetic, electrical) and construction of equivalent circuits, as well as existing approaches do not allow predicting the state of electrical systems, which would improve the efficiency and reliability of electrical substations in general.

Despite the diversity in the methods of diagnosis and control of oil transformers, the most popular are thermal imaging and transformer's oil condition monitoring method.

Thermal imaging monitoring is carried out to determine the thermal state of disconnectors, current and voltage transformers, surge arresters and surge arresters, communication capacitors, oil and air circuit breakers, busbar distributors, the quality of soldering of stator windings of turbogenerators during repair work, high-voltage bushings of power transformers, transformer cooling systems, electric motors, generators, etc. The condition of chimneys and flues at thermal power plants, the detection of places of suction of cold air, etc. are also being monitored.

Thermal imaging control in relation to power transformers is an auxiliary diagnostic method, which, along with traditional methods (measurement of insulation characteristics, open-circuit current, chromatographic analysis of gas composition in oil, etc.) provides additional information about the condition of the object. The experience of conducting IR diagnostics of power transformers showed that it can be used to identify the following malfunctions: the occurrence of magnetic fields of dispersion in the transformer due to a violation of the isolation of individual elements of the pipeline (console, studs, etc.); violation of the operation of cooling systems (oil pumps, filters, fans, etc.) and assessment of their effectiveness; a change in the internal circulation of oil in the transformer tank as a result of slag formation, structural miscalculations, swelling or displacement of the winding insulation (especially for transformers with a long service life); heating of internal contact connections of windings with transformer leads; coil closure in the windings.

The heating temperature on the surface of the oil pump housing and pipelines of a working transformer is almost the same. In the event of a malfunction in the oil pump (friction of the impellers, a short circuit in the motor winding, etc.), the temperature on the surface of the oil pump housing should increase and exceed the temperature on the surface of the oil pipe.

When analyzing thermograms, the statistical properties of the emitted surface, design features and the consideration of structural elements partially screening the control object are taken into account. Further, the information received from the thermogram is converted into a thermographic function and the defect is calculated (represented on figure 1). 


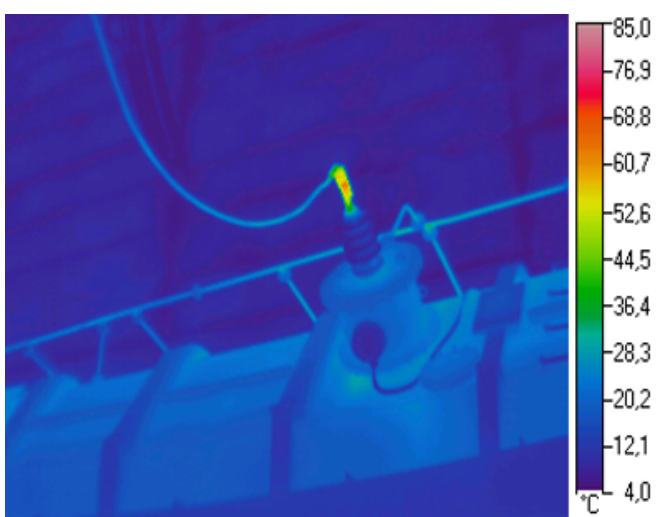

a)

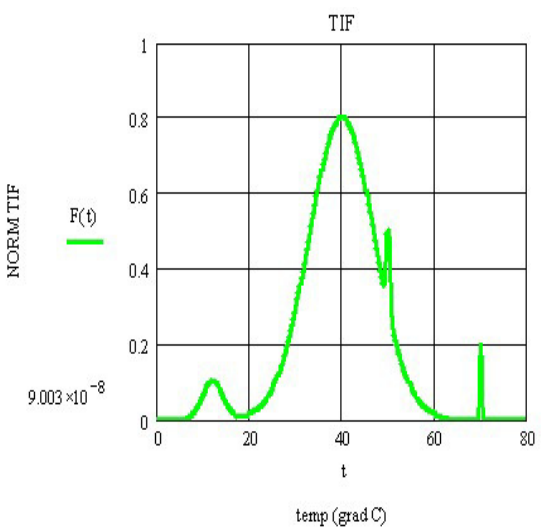

b)

Fig. 1. Illustration of the conversion of information from the thermal image "a" to the thermographic information function " $b$ " for a thermogram of a fragment of a transformer tank in the presence of a background.

Chromatographic analysis of gases dissolved in oil is a special method used to detect damage and defects in the structural components of electrical equipment, but practically does not inform about the quality and condition of the oil itself. Chromatographic analysis (HARG) allows you to:

- track the development of processes in equipment,

- identify defects at an early stage of their development that are not detectable by traditional methods,

- determine the alleged nature of the defect and the degree of damage

- navigate when determining the location of damage.

The following gases are used to assess the condition of oil-filled equipment: hydrogen $(\mathrm{H} 2)$, methane $(\mathrm{CH} 4)$, ethane $(\mathrm{C} 2 \mathrm{H} 6)$, ethylene $(\mathrm{C} 2 \mathrm{H} 4)$, acetylene $(\mathrm{C} 2 \mathrm{H} 2)$, carbon monoxide $(\mathrm{CO})$, carbon dioxide $(\mathrm{CO} 2)$. In addition, oxygen and nitrogen are always present, and their concentration varies depending on the tightness of the transformer case and gases such as propane, butane, butene and others can be released, but their study for diagnostic purposes is not widespread.

The condition of the equipment is evaluated by comparing the quantitative data obtained in the analysis with the boundary values of the gas concentration and the rate of increase in the concentration of gases in oil. It is important to distinguish between normal and excessive volumes of gas. Normal aging or gas generation varies depending on the design of the transformer, the load and the type of insulation material.

The following is the relationship between the main gases and the most characteristic types of defects (table 1).

Table 1. Interrelation of basic gases and the most typical types of defects

\begin{tabular}{|l|l|}
\hline hydrogen $\left(\mathbf{H}_{2}\right)$ & $\begin{array}{l}\text { Electric defects: partial discharges, spark and arc } \\
\text { discharges }\end{array}$ \\
\hline methane $\left(\mathbf{C H}_{4}\right)$ & $\begin{array}{l}\text { Thermal defects: heating of oil and paper-oil } \\
\text { insulation in the temperature range (400-600) }{ }^{\circ} \mathrm{C}\end{array}$ \\
& $\begin{array}{l}\text { or heating of oil and paper-oil insulation, } \\
\text { accompanied by discharges }\end{array}$ \\
\hline ethane $\left(\mathbf{C}_{2} \mathbf{H}_{\mathbf{6}}\right)$ & $\begin{array}{l}\text { Thermal defects: heating of oil and paper-oil } \\
\text { insulation in the temperature range }(300-400)^{\circ} \mathrm{C}\end{array}$ \\
\hline ethylene $\left(\mathbf{C}_{2} \mathbf{H}_{4}\right)$ & Thermal defects: heating oil and paper-oil \\
\hline
\end{tabular}




\begin{tabular}{|l|l|}
\hline & insulation above $600{ }^{\circ} \mathrm{C}$ \\
\hline acetylene $\left(\mathbf{C}_{2} \mathbf{H}_{2}\right)$ & Electric defects: electric arc, sparking \\
\hline carbon monoxide (CO) & $\begin{array}{l}\text { Thermal defects: aging and hydration of oil and / or } \\
\text { solid insulation }\end{array}$ \\
\hline carbon dioxide (CO2) & $\begin{array}{l}\text { Thermal defects: aging and hydration of oil and / or } \\
\text { solid insulation; solid insulation heating }\end{array}$ \\
\hline
\end{tabular}

Measurement of the transformer's oil (TO) impedance allows you to evaluate not only the residual life of the oil, but also the condition of the cooled surfaces, as well as deviations in the operation of the transformer. This is due to the fact that most of the malfunctions in the transformer lead to its excessive heating and, as a result, more rapid and unplanned oil degradation. Since TOs are able to accumulate electric charges during the passage of current through them, their electrical properties are not enough to describe using ohmic resistance. It is necessary to take into electrical capacitance.

The capacitance is the coefficient of proportionality between the charge and the potential and is defined as the ratio of the change in charge with $\Delta q$ of the conductor to the change in its potential $\Delta \varphi$ :

$$
C=\frac{\Delta q}{\Delta \varphi}
$$

Under equal conditions, the geometry of an object is determined by its dielectric constant, i.e. polarization phenomena. For a flat capacitor, it is determined by the formula:

$$
C=\frac{\varepsilon S}{4 \pi d}
$$

$S$ - plate area; $d$ - distance between plates.

The measured capacitance of the TO is determined by the polarization capacitance that occurs at the instant of current passage. The polarization capacitance reflects the ratio of the change in the charge of the object to the change in its potential during the passage of alternating current. Charge changes over time $t$

$$
\Delta q=\int_{0}^{t} I d t
$$

and potential change:

$$
\Delta \varphi=R\left(I_{0}-I_{t}\right),
$$

then the polarization capacitance $C_{p}$ will be determined by the following equation:

$$
C p=\frac{\int_{0}^{t} I d t}{R\left(I_{0}-I_{t}\right)},
$$

where $I_{0}$ and $I_{t}$ - initial and final values of current strength; $I$ - instantaneous current; $R$ is the resistance of the object.

When alternating current is passing through a TO, both active or ohmic $R$ and reactive or capacitive $X_{C}$ resistances arise, the total value of which is called the complex resistance, or impedance $Z$ :

$$
Z=\sqrt{R^{2}+X c^{2}}
$$

In order to more accurately diagnose transformer oil, a method of resonant seriesparallel impedancemetry (RSPI) has been developed. It combines measurements on a physical object in three modes (standard and voltage and current resonances) and solving the system of equations of the transformer oil model, which allows you to determine the equivalent circuit parameters that are inaccessible to direct measurements $[16,17]$.

The algorithm of the RSPI method contains the following steps: measurements on a physical object in three modes: standard, voltage resonance and current resonance; drawing 
up the equivalent circuit of the investigated TO; preparation and solution of the system of equations of the model relative to the desired parameters of transformer oil.

The generalized research scheme is shown in Fig. 2.

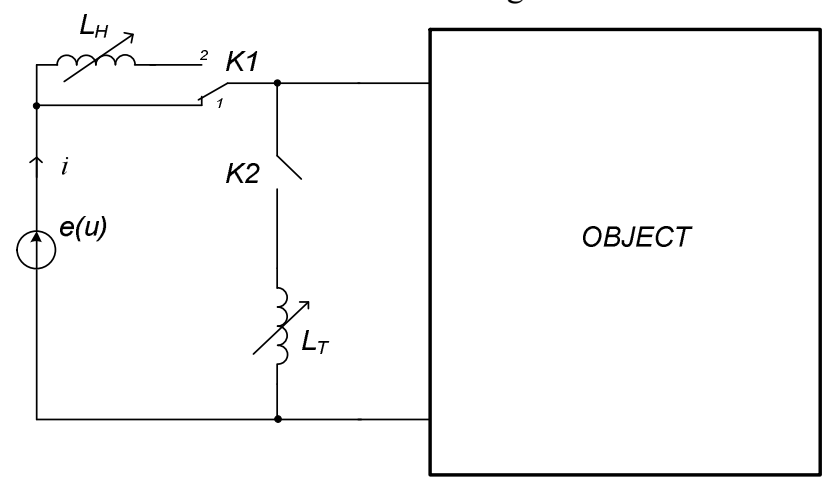

Fig. 2. Generalized experimental design

A sine voltage generator $e(u)$ is connected to the measured TO. In the normal mode (switch $\mathrm{K} 1$ is in position 1, switch $\mathrm{K} 2$ is open), the amplitude values of current I, voltage U, and phase shift $\varphi$ between them are measured. In the second mode of the measurement experiment (voltage resonance), the key $\mathrm{K} 1$ is switched to position 2, the key $\mathrm{K} 2$ is open. By changing the inductance $L_{\mathrm{H}}$ we achieve that the phase shift $\varphi$ between current $i$ and voltage $u$ is equal to zero. In this mode, the current $I=I_{\mathrm{H}}$ and voltage $U$ are measured. In the third mode of the measurement experiment (current resonance mode), key $\mathrm{K} 1$ is switched to position 1, and key $\mathrm{K} 2$ is closed. By changing the inductance $L_{\mathrm{T}}$ we achieve that the phase shift $\varphi$ between current $i$ and voltage $u$ is equal to zero. In this mode, current $I=I_{\mathrm{T}}$ and voltage $U$ are measured.

\section{$3 \quad$ Results}

The computing unit of the device for calculating the impedance $Z$ and phase $\varphi$, based on the impedance meter (Figure 3), has been developed. Functional diagram of the device is shown in Figure 4.

In fig. 3, are shown: DOSC - driving oscillator; DFS - digital frequency synthesizer; DAC - digital-to-analog converter; DFC - discrete Fourier converter; ADC - analog-todigital converter; $\mathrm{I} 2 \mathrm{C}$ - communication data bus.

The user, through the computer program, touches the desired frequency of the driving oscillator (DOSC). Next, the signal from the digital frequency synthesizer (DFS) passes through the DAC (converting the digital signal to analog), then the signal enters through a scaling buffer amplifier (SBA) and a converter amplifier (CA) (cascade with a transmission coefficient depending on the measurement of impedance $(Z)$ and feedback resistance).

$\mathrm{CA}$ amplifies the voltage to the value necessary for the 12-bit ADC to operate. The code generated at the ADC output is stored in a buffer register designed to store its 1024 values. The discrete Fourier converter (DFC), calculates the real and imaginary components of the impedance, the results of which are transmitted to the microcontroller, which performs the following main functions:

- frequency synthesizer control;

- control of the amplifier-converter to maintain the signal at its output in the working range of the $\mathrm{ADC}$;

- scaling buffer amplifier control; 
- calculation of the module and phase of the impedance based on the results of the DFC;

- implementation of data exchange with a computer, indicator and keyboard.

In this case, the signal supplied to the microcontroller is fed to the impedance and frequency computing unit.

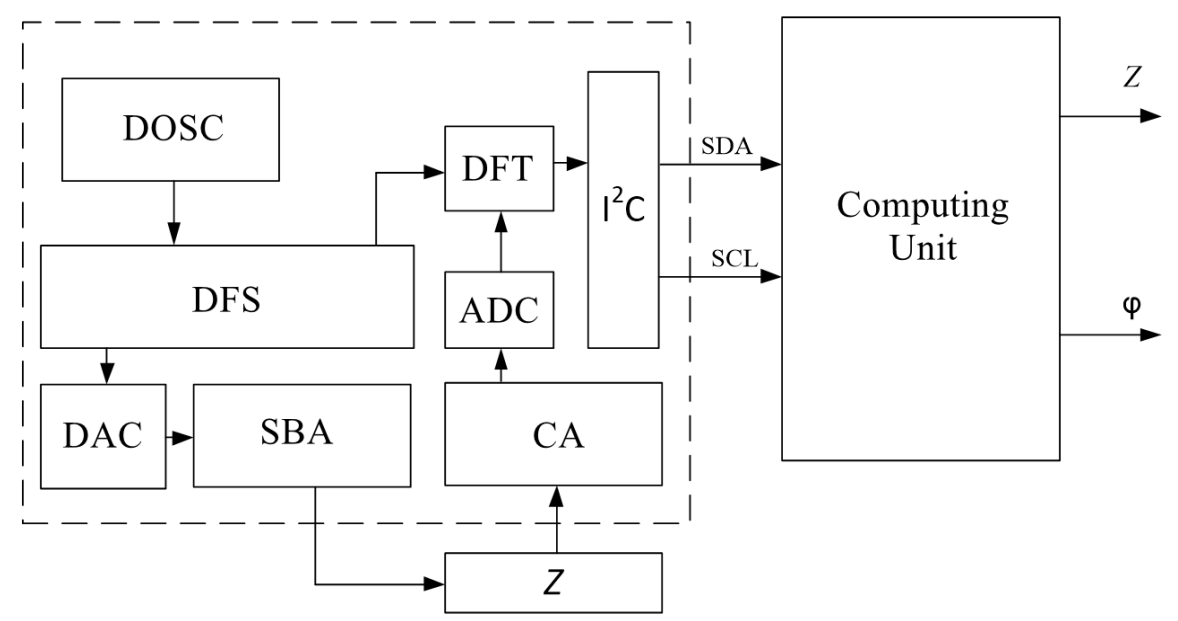

Fig. 3. Block diagram of the impedance meter

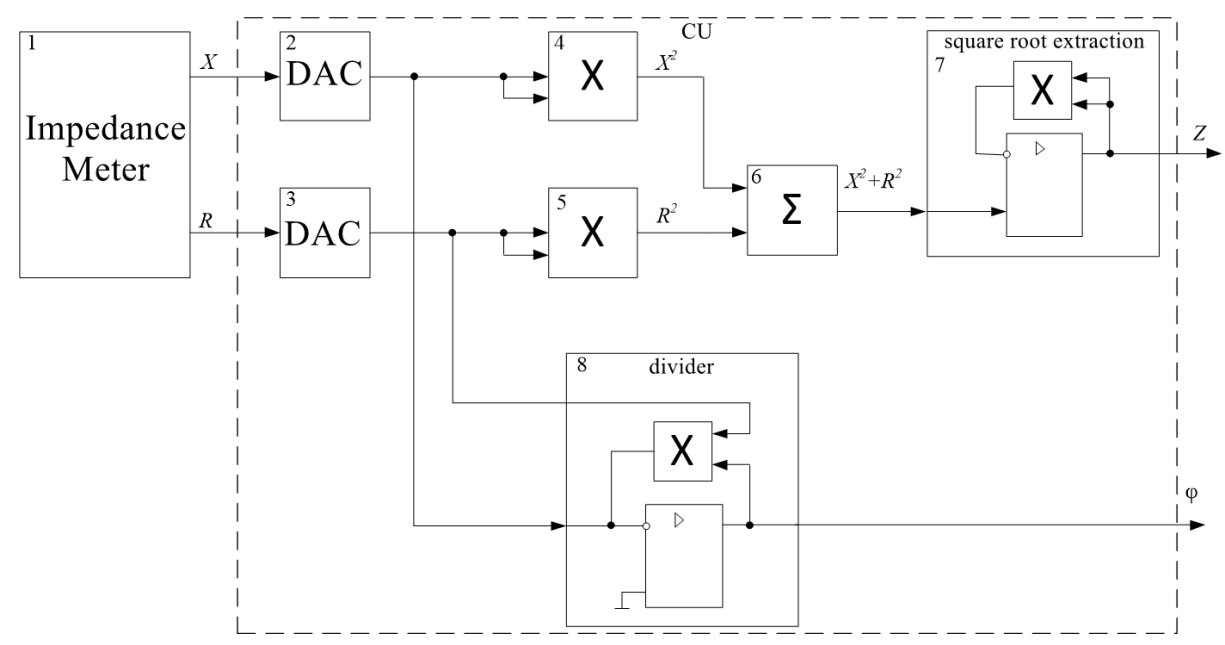

Fig. 4. Functional diagram of a computing device

Figure 4 shows: 1 - impedance meter [18], two signals are sent from its output to the computing unit $(\mathrm{CU})$ : it's an active $(R)$ and reactive $(X)$ components of the impedance. Blocks 2 and 3 are digital-to-analog converters (DACs), from the outputs of which an analog signal is fed to the multipliers. In these blocks, the analog signals are squared and then block 6 (built on the op-amp) are summed. Block 7 is a square root calculation scheme, here a signal multiplier is included in the feedback of the op-amp. Block 8 is a divider constructed in the same way on an op-amp and with a multiplier included in the feedback.

The simulation of the computing unit will be using the LabVIEW graphical development environment. 
LabVIEW (Laboratory Virtual Instrumentation Engineering Workbench) is a development environment and platform for executing programs created in the graphical programming language «G».

Let use formulas to calculate the modulus and impedance phase. The block diagram of the program is shown in Figure 4, the front panel of the program in Figure 5.

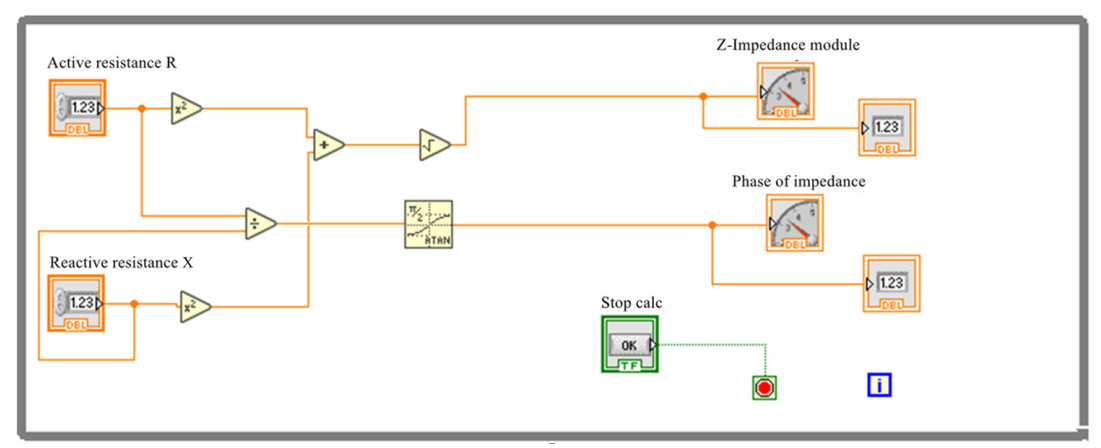

Figure 4-Block diagram of the program.

Values of active resistance is set to $R=3$ and reactive is set to $X=2$, and taken results are shown on Figure 5.

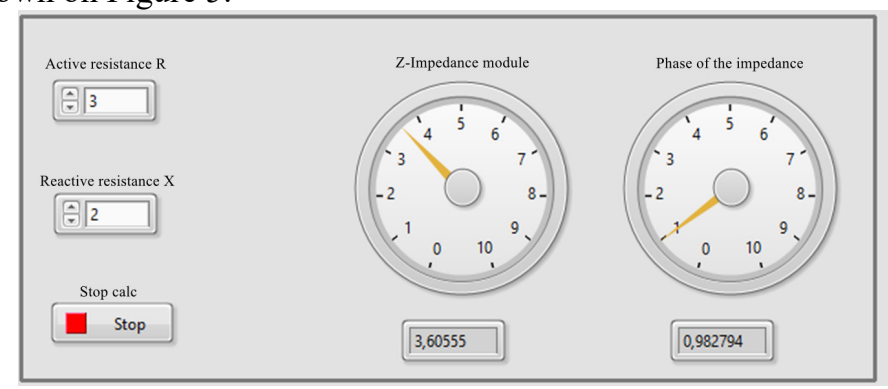

Fig. 5. Indications on the front of the program.

\section{Discussion}

Of the entire spectrum of existing methods for diagnosing and monitoring the functional state of power oil transformers in electrical substations, the impedance method is noteworthy. It guarantees high measurement accuracy, allows for diagnosis to identify the development of the resource of transformer oil, as well as assess the significance of factors affecting the accelerated deterioration of the state of the oil.

\section{Conclusion}

As a result, we examined the existing fault diagnosis of power oil transformers, on the basis of which we can conclude that the methods currently used have a number of disadvantages that impede frequent technical control. In this regard, the authors proposed a method of impedance serial-parallel identification, and also developed electrical circuits of a device that implements this approach.

\section{Acknowledgements}


This article has been prepared as a result of the implementation of project № SP1544.2019.1, implemented under the program "Scholarships of the President of the Russian Federation to young scientists and graduate students carrying out promising research and development in priority areas of modernization of the Russian economy, for 2019-2021 ". This article was prepared using the equipment of the CUC "Diagnostics and energyefficient electrical equipment" of SRSPU (NPI).

\section{References}

1. N.V. Kinsht, Vologda Readings 53, 13 - 14 (2003)

2. L.V. Makarevich, L.N. Shifrin, M.E. Alpatov, University News. Mechanical Engineering, 155-159 (2012)

3. V.M. Stepanov, K.A. Andreev, Bulletin of Tula State University, Technical science, 74 $-81(2011)$

4. E.G. Ermakov, T.V. Ganul, Electro, Electrical engineering, electric power industry, electrical industry, $32-36$ (2015)

5. Purwadi, N. Heryana, D. Nurafiat, C. Sosetyo, A. Setiana and A. Mustaqim, Proceedings of the 2011 International Conference on Electrical Engineering and Informatics, Bandung, 1-5 (2011)

6. Richardson, IEE Colloquium on Condition Monitoring of Large Machines and Power Transformers (Digest No: 1997/086), 6/1-6/2, London, UK (1997)

7. Y. Perkasa, N. Lelekakis, J. Wijaya and D. Martin, 22nd Australasian Universities Power Engineering Conference (AUPEC), 1 - 5 (2012)

8. Mackenzie, J. Crossey, A. dePablo and W. Ferguson, 2010 IEEE International Symposium on Electrical Insulation, 1-5, San Diego, CA (2010)

9. Zmarzly, T. Boczar, P. Fracz and S. Borucki, 2014 IEEE International Power Modulator and High Voltage Conference (IPMHVC), 561-564, Santa Fe, NM, (2014)

10. B.A. Alekseev, Large power transformers: state monitoring at work and during revision. Energoprogress, Moscow (2010)

11. Yu.P. Aksenov, Guidelines for the diagnosis of power transformers, autotransformers, shunt reactors and their inputs. Diagnostic Complexes and Systems LLC (2006)

12. M. Ershov, Art of circuitry, 6 - 10 (2007)

13. L. Shang and W. Liang, 5th International Conference on Electric Utility Deregulation and Restructuring and Power Technologies (DRPT), 1772-1775, Changsha (2015)

14. V. Z. Manusov, D. V. Orlov and V. V. Frolova, 2018 IEEE International Conference on Environment and Electrical Engineering and 2018 IEEE Industrial and Commercial Power Systems Europe (EEEIC / I\&CPS Europe), 1-6, Palermo (2018)

15. I. Khalyasmaa, N. A. Babushkina, S. A. Dmitriev and D. A. Glushkov, International Conference on Computer Technologies in Physical and Engineering Applications (ICCTPEA), 72-72, St. Petersburg (2014)

16. G.I. Tkachenko; A.M. Lankin, M.V. Lankin, 2nd International Conference on Industrial Engineering, Applications and Manufacturing (ICIEAM), 2016

17. G.I. Tkachenko, A.N. Baklanov, International Conference on Industrial Engineering Procedia Engineering, 690 - 694 (2015)

18. S. Obraztsov, Yu. Troitsky, Modern Electronics, 12 - 15 (2009) 